\title{
A Prospective Study on Factors Affecting Morbidity in Patients Undergoing Emergency Abdominal Surgery
}

\author{
Jyothi J.1 ${ }^{1}$, Sharanbasappa Bellikatti ${ }^{2}$, Chetan Channabasawaraj Padashetty ${ }^{3}$ \\ 1,2,3 Department of General Surgery, Basaveshwara Teaching and General Hospital \\ Attached to Mahadevappa Rampure Medical College Kalaburagi, Karnataka, India.
}

\section{ABSTRACT}

\section{BACKGROUND}

Emergency surgery is defined as a non-elective surgery that is performed with the aim to prevent morbid or fatal health consequences of a surgically treatable disease. Emergency surgical procedures, account for a small fraction of all surgical procedures, but for a disproportionately large amount of postoperative morbidity and mortality. Abdominal emergency surgery has adverse effects on the results of surgery. Predictors of higher mortality and morbidity for emergency abdominal surgery were defined. The predictors of post-operative complications of abdominal emergency surgery have been identified. The independent predictors of morbidity were blood urea, total protein, sex, smoking, serum creatinine, age, serum albumin and procedure duration.

\section{METHODS}

We have reviewed patients who had undergone an abdominal emergency surgery between October 2016 and August of 2018 at Basaveshwara Teaching and General Hospital at MR Medical College, Kalaburagi. Especially those who had undergone abdominal emergency surgery within 12 hours of admission to the hospital. Our primary outcome was postoperative complications seen in patients within 30 days.

\section{RESULTS}

58 percent of 50 patients had experienced one or more than one complication with a mortality of 6 percent within 30 days. Respiratory and SSI (surgical site infections) were the most commonly seen complications.

\section{CONCLUSIONS}

Patients with postoperative complications who had undergone emergency abdominal surgery probably are aged people, patients with elevated levels of creatinine \& blood glucose and males with reduced levels of serum albumin, smokers and males who had longer procedure duration. Fluid resuscitation and experienced surgeons are important to enhance results.

\section{KEY WORDS}

Abdominal Surgery, Emergency, Post-Operative Complications, Morbidity Factors
Corresponding Author: Dr. Jyothi J.,

Basaveshwar Teaching and General Hospital Attached to Mahadevappa Rampure Medical College,

Kalaburagi, Karnataka, India.

E-mail: drjyothi115@gmail.com

DOI: $10.14260 /$ jemds/2021/57

How to Cite This Article: Jyothi J, Bellikatti S, Padashetty CC. A prospective study on factors affecting morbidity in patients undergoing emergency abdominal surgery. J Evolution Med Dent Sci 2021;10(05):253-257, DOI: $10.14260 /$ jemds $/ 2021 / 57$

Submission 28-09-2020,

Peer Review 22-11-2020,

Acceptance 28-11-2020,

Published 01-02-2021.

Copyright (C) 2021 Jyothi J. et al. This is an open access article distributed under Creative Commons Attribution License [Attribution 4.0 International (CC BY 4.0)] 


\section{BACKGROUND}

The emergency surgery is described as non-elective surgery, which is carried out to prevent a disease that is surgically treatable from having a fatal or morbid health effect.1,2 Emergency surgery procedures account for a small fraction of all surgical procedures, but a disproportionately large amount of postoperative morbidity and mortality. Every year millions of individuals worldwide need immediate time-critical abdominal emergency operations to overcome the potentially fatal obstruction of small bowels, haemorrhage, perforation of the gastrointestinal tract, invasive cancer tumours, injuries of blunt force / penetrative trauma and peritonitis. ${ }^{3}$ Emergency surgery is one case where a surgeon faces a therapeutic challenge. It is obvious that in such situations, majority of the patients have a low life span so surgical procedure seems inevitable. Post-operative results are usually worse following emergency abdominal surgeries compared to elective surgery.

In the last decade, major emphasis has been put on decreasing postoperative complications. Despite the emphasis on improved surgery efficiency, studies consistently show that emergency conditions make an important contribution to mortality and morbidity in abdominal surgery patients. 4,5 Advanced age and perioperative complications such as sepsis and functional condition lift the mortality rate to more than 50 percent. ${ }^{6}$ The organisation of emergency departments is varying considerably ${ }^{7,8}$ and there is little evidence for effective treatment of emergency surgery patients. ${ }^{9,10}$ A significant group of patients, who are experiencing a broad variety of illnesses and current comorbidities, is the general surgical group. The outcomes of this patient group may vary.

Bowel obstruction, bleeding, perforated peritonitis and appendicitis are the prime emergency abdominal surgical presentations. Patients with postoperative complications with emergency abdominal surgery probably are smokers, males and are aged, who have higher perioperative blood glucose, lower levels of serum albumin and creatinine level. ${ }^{11}$ The patients with these complications acquire longer surgical duration. ${ }^{7}$ Pre-operative intravenous fluid treatment to sufficiently revive patients' closer monitoring of glucose, and trained operating teams to minimize procedure duration is expected to improve outcomes in emergency abdominal surgery patients.

\section{Objectives}

- To determine the factors that contribute to morbidity associated with patients undergoing emergency abdominal surgery.

- To identify the risk factors that are potentially modifiable, which reduce post-operative complications of emergency abdominal surgery.

\section{METHODS}

A prospective study of 50 patients undergoing emergency abdominal surgery was done from October 2016 till August of 2018. The duration of study was 2 years. In Basaveshwara, Teaching \& General Hospital, Kalaburagi, patients who underwent abdominal emergency surgery over two years were included in the study. The history of patients was noted.
Acute Physiologic Assessment and Chronic Health Evaluation (APACHE) score calculation was done. The following were included as post-operative complications: surgical site infection (deep, superficial, organ spacing), bleeding, sepsis, urinary (infection of the urinary tract or acute renal failure), cardiac, respiratory (pneumonia or pulmonary embolism), deep vein thrombosis (DVT).

It is commonly classified into two categories including the patient with complications and with no complications, with following factors: transmissible diseases (from other hospital or home), use of steroids, diabetes mellitus, wound class, sex, American Society of Anaesthesiologists (ASA) class, chronic obstructive pulmonary disease (COPD), deep vein thrombosis (DVT) prophylaxis, smoking, antibiotic administration and sepsis.

\section{Inclusion Criteria}

The patients who had undergone emergency abdominal surgery after admitting to the hospital within 12 hours, over a period of two years, in Basaveshwara Teaching \& General Hospital, Kalaburagi.

\section{Exclusion Criteria}

Those patients who for some reason quit the study. Patients of less than twelve years of age.

Patients who had prior surgery within 30 days.

Patients who were immunocompromised.

\section{Statistical Analysis}

In the current study, the inferential and descriptive statistical analysis was done. Statistical Package for the Social Sciences (SPSS) version 18 International Business Machines (IBM) Corporation, SPSS Inc., Chicago, IL, U.S.A.) has been used to analyse these findings. Mean \pm SD (min-max) was presented with results from continual measurements and results were presented with frequency (percentage) on categorical measurements.

\section{RESULTS}

\section{Age Distribution}

The distribution of patients in our analysis is between the age of 17 and 85 years. 19 patients were 55 years of age and more postoperative complications were observed in elderly patients as compared to young patients who underwent emergency surgery.

\section{Sex Distribution}

There was a total of $34(68 \%)$ male patients and $16(32 \%)$ females, thus the number of male patients was comparatively high. The chi-square value was of 0.61 and was not statistically significant, so postoperative morbidity concerning sex is not significant. 


\section{Smoking History with Postoperative Complications \\ Our analysis included 26 smokers, 22 of whom had postoperative complications that demonstrated a stronger correlation between the history of smoking and postoperative complications.}

\section{Occupation}

In our study 12 were coolie by occupation, 11 businessmen, 15 farmers and 12 housewives. The patients belonged to low to the medium socioeconomic group.

\section{Diagnosis}

18 patients with perforation (gastroduodenal, ileal), 4 patients suffering from little bowel obstruction, 7 patients with gangrene of jejunum and ilium respectively. 3 patients with acute appendicitis and 7 with strangulated inguinal hernia.

\begin{tabular}{|ccc|}
\hline Diagnosis & No of Cases & Percentage \\
\hline Ileal perforation & 11 & 22 \\
Haemoperitoneum & 10 & 20 \\
Gangrenous ileum & 7 & 14 \\
Duodenal perforation & 6 & 12 \\
Gastric perforation & 1 & 2 \\
Strangulated inguinal hernia & 7 & 14 \\
Obstruction due to adhesions & 4 & 8 \\
\hline Intussusception & 1 & 2 \\
\hline Acute appendicitis & 3 & 4 \\
\hline & Table 1. Diagnosis \\
\hline
\end{tabular}

\begin{tabular}{|ccc|}
\hline Parameters & Mean & S.D. \\
\hline HB \% & 10.88 & 2.18 \\
RBS & 144.28 & 50.7 \\
\hline Blood urea & 45.62 & 22.08 \\
Serum creatinine & 1.49 & 0.57 \\
\hline Total protein & 4.84 & 0.95 \\
\hline Serum albumin & 2.572 & 0.795 \\
\hline Table 2. Pre-Operative Biochemical Parameters \\
\hline
\end{tabular}

\section{Measure of Postoperative Complications}

50 patients in our study had undergone abdominal emergency surgery, and post-operative complications were seen in 29 of them.

\section{Anaemia and Post-operative Complications}

Anaemia is described as $\mathrm{Hb} \%<11$ gm / dL. In our analysis, the value of chi-square was 22.55 and the value of $\mathrm{p}$ was < 0.0000 which indicates that there is a greater correlation of $\mathrm{Hb}$ $\%$ and postoperative complications. Furthermore, the above table indicates that with lower $\mathrm{Hb} \%$ value postoperative complications are greater.

\begin{tabular}{|ccccc|}
\hline \multicolumn{4}{|c|}{ Complications } \\
Parameters & Yes N = 29 (58 \%) & No N = 21 (42 \%) & $\begin{array}{c}\text { Statistical } \\
\text { Analysis }\end{array}$ \\
\hline $\mathrm{HB} \%$ & $<11$ & $25(86 \%)$ & $04(19 \%)$ & $\chi 2=22.55$, \\
$(\mathrm{gm} / \mathrm{dL})$ & $>11$ & $04(14 \%)$ & $17(81 \%)$ & $\mathrm{p}<0.000$ \\
\hline \multicolumn{3}{|c|}{ Table 3. Hb \% and Post-Operative Complications } \\
\hline
\end{tabular}

\section{RBS and Post-Operative Complications}

The normal level of random blood sugar (RBS) is $180 \mathrm{mg} / \mathrm{dL}$. Hyperglycaemia is when RBS is greater than $180 \mathrm{mg} / \mathrm{dL}$. 30 patients in our analysis had hyperglycaemia, 25 of them have postoperative complications, the value of chi-square was 19.75 and the value of $\mathrm{p}$ was $<0.000$; therefore, there is a higher correlation between postoperative complications and hyperglycaemia.

\section{Blood Urea Levels and Post-operative Complications}

Table 2 illustrates that in 31 patients, the blood urea level is increased, and 25 of them have postoperative complications. The value of chi-square is 17.17 and the value of $p$ is $<0.000$, thus a higher correlation between postoperative complications and elevated blood urea is noted.

\section{Serum Creatinine Levels and Post-operative Complications}

In our analysis, there were 32 patients who had elevated creatinine levels i.e., > $1.4 \mathrm{mg} / \mathrm{dL}, 26$ of them had postoperative complications. The value of chi-square was 19.72 and the value of $p$ was $<0.000$, thus there is a higher correlation between postoperative complications and elevated creatinine levels.

\begin{tabular}{|cccc|}
\hline Parameters & \multicolumn{2}{c|}{$\begin{array}{c}\text { Complications } \\
\text { Yes N = 29 (58 \%) }\end{array}$} & $\begin{array}{c}\text { So N = 21 (42 \%) } \\
\text { Analysis }\end{array}$ \\
$\begin{array}{c}\text { Serum } \\
\text { albumin } \\
(\mathrm{mg} / \mathrm{dL})\end{array}$ & $27(93 \%)$ & $12(57 \%)$ & \\
\hline \multicolumn{2}{|c|}{ Table 4. Serum Albumin and Post-Operative Complications } \\
\hline
\end{tabular}

In our analysis, there were 39 patients who had elevated albumin i.e., > $1.4 \mathrm{mg} / \mathrm{dL}, 27$ of them had post-operative complications. The value of chi-square was 9.17 and the value of $\mathrm{p}$ was $<0.000$, thus there is a significant correlation between postoperative complications and elevated albumin levels.

\begin{tabular}{|cccc|}
\hline $\begin{array}{c}\text { Procedure } \\
\text { Duration }\end{array}$ & Yes N = 29 (58 \%) & No N = 21 (42 \%) & $\begin{array}{c}\text { Statistical } \\
\text { Analysis }\end{array}$ \\
$<60 \mathrm{~min}$ & $2(7 \%)$ & $3(14 \%)$ & \\
$60-90 \mathrm{~min}$ & $7(24 \%)$ & $1(5 \%)$ & $\chi 2=8.836$, \\
$90-120 \mathrm{~min}$ & $12(41 \%)$ & $4(19 \%)$ & $\mathrm{P}=0.0315$ \\
$>120 \mathrm{~min}$ & $8(28 \%)$ & $13(62 \%)$ & \\
\hline \multicolumn{4}{|c|}{ Table 5. Duration of the Procedure (in Minutes) } \\
\hline
\end{tabular}

The value of chi-square was 8.836, the value of $\mathrm{p}$ was 0.0315 , thus there is a significant correlation between postoperative complications and procedure duration. It is noticeable from the table 5 that if the procedure is longer then there will be more post-operative complications.

\begin{tabular}{|cccc|}
\hline $\begin{array}{c}\text { Diabetes } \\
\text { Mellitus }\end{array}$ & \multicolumn{2}{c|}{ Complications } & $\begin{array}{c}\text { Statistical } \\
\text { Analysis }\end{array}$ \\
Yes & $17(59 \%)$ & No N = 21 (42 \%) & \\
No & $12(41 \%)$ & $3(14 \%)$ & $\chi 2=9.97$ \\
\hline \multicolumn{4}{|c|}{$\begin{array}{l}\text { Table 6. History of Diabetes Mellitus and } \\
\text { Post-Operative Complications }\end{array}$} \\
\hline
\end{tabular}

In our study 20 patients were diabetic, 17 of them had postoperative complications. The value of chi-square was 9.97, 
the value of $\mathrm{p}$ was 0.0015 , and thus, there is a significant correlation between postoperative complications and diabetes mellitus.

\section{Types of Complications}

There were 12 patients with surgical site infection (SSI), 4 patients with acute renal failure (ARF), 9 patients with multiorgan dysfunction syndrome (MODS), and 1 patient with myocardial infarction (MI).

\section{DISCUSSION}

50 patients were evaluated in our study, 34 (68\%) were males and 16 (32\%) were females. $42 \%$ were males and $58 \%$ were females in the analysis of Akinbami et al. ${ }^{1}$

In our analysis, hernia surgery 7 (14\%), appendectomy 3 (6\%), haemoperitoneum due to trauma abdomen $10(20 \%)$, gastro-duodenal ulcer perforation closure 7 (14 \%), laparotomy with adhesiolysis 4 (8\%), ileal perforation closure 11 (22\%), were the patients who had undergone abdominal emergency surgery. Most of the patients underwent surgery for ileal perforation and haemoperitoneum due to trauma abdomen.

In our analysis, 29 (58\%) patients had post-operative complications, however, there were 202 (24.7\%) cases in Akinbami et al. ${ }^{1}$ who had postoperative complications after thirty days of intervention. The mortality rate in our analysis was $3(6 \%)$ and $73(8.9 \%)$ in Akinbami et al. ${ }^{1}$ within thirty days of intervention. Surgical site infections as well as respiratory complications were the main postoperative complications. Mortality was due to acute renal failure (ARF), myocardial infarction (MI), multiple organ dysfunction syndromes (MODS) and acute respiratory distress syndrome.

The majority of the patients undergoing surgery in emergency included 31 (62\%) patients under the age of 55 and 19 (38\%) patients over the age of 55. Seventeen patients (89\%) were seen with post-operative complications and mortality in $2(10 \%)$ out of 19 patients whose age was above 55 years. As age increases, postoperative morbidity increases.

The postoperative complications were seen in $72 \%$ male and $28 \%$ female patients who had undergone abdominal emergency surgery and morbidity is more apparent in male patients in our analysis. The reason has yet to be determined.

26 patients were smokers, 22 of them had postoperative complications. Causes of smoking limit collagen synthesis and reduces neutrophils' oxidative killing function. Therefore, postoperative complications affect smokers more. ${ }^{12}$

The detrimental effect on injury recovery is considered to be hyperglycaemia. Only 30 patients in our analysis were suffering from hyperglycaemia and 25 of them had postoperative complications. Effective regulation of glycaemia lowers postoperative complications.

Elevated blood urea is when the level of blood urea is above $45 \mathrm{mg}$ / dL; there were 31 patients who had an elevated level of blood urea and post-operative complications were seen in 25 patients. The value of chi-square was 17.17 and the value of $p$ was 0.00003 . Thus, the correlation between postoperative complications and blood urea level is higher. An elevated level of blood urea, perhaps because of hypovolemia, in our analysis, may help patients revive intravenous fluid sufficiently. Due to this, the post-operative complications were decreased.

The serum creatinine level above $1.4 \mathrm{mg} / \mathrm{dL}$ is considered to be an increased level of serum creatinine. There were 32 patients who had increased levels of serum creatinine, and post-operative complications were seen in 26 patients. The value of chi-square was 19.72 and the value of $p$ was 0.000008 . Thus, there is a higher correlation between postoperative complications and an increased level of serum creatinine.

Total protein and reduced levels of serum albumin have a significant correlation with postoperative complications (value of $p$ was 0.002 ). When the level of serum albumin was below 3 then the patients had more postoperative complications, in Golub's et al. analysis. Post-operative changes are treated by supplying intravenous albumin supplementation or oral protein supplementation. ${ }^{13}$

The postoperative complications are also increased due to surgery duration. So the team of doctors performing the surgery should be well trained to reduce the duration of surgery. In addition to this, the latest assistance to acute care surgeons coping with trauma and emergency general surgery is an approach that has to be more extensively studied. Through their experience, acute care surgeons can minimize surgical durations and delays. ${ }^{14}$

In surgical procedures, anaesthesia procedures as well as postoperative patient treatment substantial improvement has been achieved concerning outcomes of surgery in the geriatric population; because of their depleted physiological reserves, surgery remains a prime reason for mortality and morbidity among aged patients.

It is an attempt to reduce the mortality rate. We must take precautions to prevent complications seen in geriatric patients with a higher mortality rate seen in later stages of complications. For example, the aged patients who have been suffering from common diseases like acute cholecystitis or incarcerated hernia may be given elective surgery to prevent an emergency procedure. The organized control of the disease would make surgery more secure.

To minimize delay in surgery through daytime operating rooms and surgeon's experience to enhance the patient decision-making, Britt et al. have designed an emergency surgery model. 15

Earley et al. analysed the results of this model for patients with appendectomy and observed a reduction in surgery time, stay length and complication rate. ${ }^{14}$

\section{CONCLUSIONS}

In patients who had undergone abdominal emergency surgery, post-operative complications most probably developed in patients who had elevated levels of blood urea and preoperative serum creatinine, lower level of serum albumin, or in males who are smokers and in elderly people.

Those patients who had postoperative complications also had a longer duration of stay in the hospital. This is a financial burden to the patients.

Preoperative administration of intravenous fluid to appropriately revive patients, sufficient glucose control, and trained and experienced operating team to minimize 
procedure duration are expected to enhance results of abdominal surgery in an emergency.

In an attempt to reduce the mortality rate, we must take precautions in advance to prevent complications seen in geriatric patients with a higher mortality rate seen in later stages of complications. For example, the aged patients who have been suffering from common diseases like acute cholecystitis or incarcerated hernia.

Prior to surgery, the risk factors associated with the patient must be evaluated and immediately rectified. An experienced surgeon should operate on patients with high risk. The postoperative care of patients is very important. When risk factors can be identified earlier, their numbers can be minimized, and the occurrence of postoperative complications will certainly decrease.

Data sharing statement provided by the authors is available with the full text of this article at jemds.com.

Financial or other competing interests: None.

Disclosure forms provided by the authors are available with the full text of this article at jemds.com.

\section{REFERENCES}

[1] Akinbami F, Askari R, Steinberg J, et al. Factors affecting morbidity in emergency general surgery. Am J Surg 2011;201(4):456-62.

[2] American College Of Surgeons National Surgical Quality Improvement Programme (ACS NSQIP). ACSNQIP variables and definition. Chapter - 4. 2018. http://acsnqip.org/.

[3] Stewart B, Khanduri P, McCord C, et al. Global disease burden of conditions requiring emergency surgery. Br J Surg 2014;101(1):e9-22.

[4] Bratzler DW. The surgical infection prevention and surgical care improvement projects: promises and pitfalls. Am Surg 2006;72(11):1010-6.

[5] Khuri SF, Daley J, Henderson WG. The comparative assessment and improvement of quality of surgical care in the department of veterance affairs. Arch surg 2002;137(1):20-7.

[6] Al-Temimi MH, Griffee M, Enniss TM, et al. When is death inevitable after emergency laparotomy? Analysis of the American College of Surgeons National Surgical Quality Improvement Program Database. J Am Coll Surg 2012;215(4):503-11.

[7] Dimick JB, Chen SL, Taheri PA. Hospital costs associated with surgical complications: a report from the private sector National Surgical Quality Improvement Program. J Am Coll Surg 2004;199(4):531-7.

[8] Ramos M, Khalpey Z, Lipsitz S, et al. Relationship of perioperative hyperglycemia and postoperative infections in patients who undergone general and vascular surgery. Ann surg 2008;248(4):585-91.

[9] Gibbs J, Cull W, Henderson W, et al. Pre-operative serum albumin level as a predictor of postoperative mortality and morbidity: results from the National VA Surgical Risk Study. Arch Surg 1999;134(1):36-42.

[10] Costa G, Tomassini F, Tierno SM. Emergency colonic surgery: analysis of risk factors predicting morbidity and mortality. Chir Ital 2009;61(5-6):565-71.

[11] Khoja HR, Garg D, Gupta M, et al. Evaluation of risk factors and outcome of surgery in elderly patients. J Indian Acad of Geriat 2008;1:14-17.

[12] Brown RO, Bradley JE, Bekemeyer WB, et al. Effect of albumin supplementation during parenteral nutrition on hospital morbidity. Crit Care Med 1988;16(12):1177-82.

[13] Lewis MC. Physiologic changes in the elderly. University of Miami School of Medicine. https://pogoe.org/sites/default/files/gsr/2_Physiologic _Changes_In_The_Elderly.pdf

[14] Dagla R, Kumar P. A prospective study of factors affecting post-operative morbidity and mortality following abdominal surgery in elderly. Indian Journal of Applied Research 2018;8(1):207-10.

[15] Svenningsen P, ManoharanT, Foss NB, et al. Increased mortality in the elderly after emergency abdominal surgery. Dan Med J 2014;61(1):A4876. 